\title{
Andere Zeiten, andere Inhalte. Bedeutungsgehalt und Bedeutungs- wandel der politischen Richtungsbegriffe Links und Rechts im innerdeutschen Vergleich*
}

\author{
Eva-Maria Trüdinger und Uwe Bollow
}

Bis heute spielt der Links-Rechts-Gegensatz eine wichtige Rolle in den öffentlichen Diskursen in Deutschland. Die beiden Begriffe Links und Rechts strukturieren seit der Französischen Revolution den politischen Raum. In der Nationalversammlung gruppierten sich die Befürworter einer neuen Ordnung auf der linken Seite und die Kräfte, die an der bisherigen Monarchie festhalten wollten, rechts im Parlament. ${ }^{1}$ Diese ideologischen Etiketten fanden bald Verbreitung über Frankreich hinaus und dienen seither als politische Richtungsbegriffe und Instrument der politischen Kommunikation, um Positionen der politischen Akteure aufzuzeigen und die Unterstützung der Wählerschaft zu mobilisieren. Über diese Instrumente wird zwischen politischen Eliten und Wählern kommuniziert, etwa wenn wie im Superwahljahr 2009 die Strategen der Parteien diese Begriffe benutzen, mit Inhalten verbinden und über linke und rechte Positionen der Parteien und den Platz in der Mitte streiten. Im Sinne von symbolisch generalisierten Kommunikationsmedien ${ }^{2}$ lassen sie sich demnach im politischen System als wichtiges Verbindungsglied in der Kommunikation zwischen Bürger und politischen Eliten modellieren. Außerdem sind Links und Rechts für die Bevölkerung Orientierungshilfen und Urteilsheuristiken in einer komplexen politischen Welt. ${ }^{3}$ Sie können als Entscheidungshilfe dienen und dafür sorgen, dass sich die Bürger trotz fehlender Informationen eine Meinung über ein Thema bilden können.

Die Untersuchung der Frage, was die deutsche Bevölkerung unter Links und Rechts versteht, ist in mehrerlei Hinsicht von Bedeutung und soll im Mittelpunkt dieses Beitrags stehen: Wie die Bürger Links und Rechts in ihrer Meinungsbildung nutzen, hängt davon $\mathrm{ab}$, wie präsent diese sind und welchen Orientierungen sie nahestehen. Und wenn die Kommunikation zwischen politischen Eliten und Bürgern über diese Etiketten passiert, dann ist es wichtig, dass ähnliche Inhalte mit diesen Begriffen verknüpft werden ${ }^{4}$ und dass bekannt

* Für Hilfestellungen und Hinweise zu den früheren Auswertungen, auf die wir Bezug nehmen, danken wir Peter Selb, Petra Bauer-Kaase und Jan van Deth, für hilfreiche Kommentare zum Aufsatz Oscar W. Gabriel.

1 Vgl. Jean A. Laponce, Left and Right: The Topography of Political Perceptions, Toronto / Buffalo / London 1981, S. $48 \mathrm{ff}$.

2 Vgl. Niklas Luhmann, Soziologische Aufklärung 2. Aufsätze zur Theorie der Gesellschaft, Opladen 1975, S. 174.

3 Vgl. Dieter Fuchs / Hans-Dieter Klingemann, The Left-Right Schema, in: M. Kent Jennings / Jan van Deth, (Hrsg.), Continuities in Political Action. A Longitudinal Study of Political Orientations in Three Western Democracies, Berlin / New York 1990, S. 203 - 234.

4 Vgl. Susumu Shikano / Franz Urban Pappi, The Positions of Parties in Ideological and Policy Space: The Perception of German Voters of their Party System, in: Working Papers, Mannheimer Zentrum für Sozialforschung, Nr. 73, Mannheim 2004, S. 2. 
ist, welches Verständnis von Links und Rechts in der Bevölkerung vorherrscht. Es besteht also Forschungsbedarf zu den Inhalten, die die Bürger mit Links und Rechts verknüpfen, vor allem weil die damit verbundenen Vorstellungen der Individuen als ideologische Dispositionen - zahlreichen empirischen Analysen zufolge - auch erheblichen Einfluss auf verschiedene Dimensionen politischer Einstellungen und politischen Verhaltens haben. ${ }^{5}$ In diesem Zusammenhang wird in der Forschung eine weitere Frage diskutiert, auf die hier aktuelle Antworten gegeben werden sollen. Wenn Links und Rechts Orientierung für die Bürger in politischen Auseinandersetzungen liefern sollen und sich diese Konflikte, deren Themen und Akteure verändern, dann kann die Untersuchung des Bedeutungswandels im Sinne eines Wandels $s^{6}$ von zugeschriebenen Inhalten Aufschluss über die Flexibilität des Links-Rechts-Schemas in Deutschland geben. Insbesondere interessiert dabei, wie präsent bei den Bürgern heute noch diejenigen Assoziationen sind, die schon früh ${ }^{7}$ als zentrale Inhalte von Links und Rechts genannt wurden, wie etwa Positionen zum sozialen Wandel oder zu Verteilungsfragen. Bisher wurde die Veränderung in der Bedeutung der LinksRechts-Dimension meist vor dem Hintergrund eines Wertewandels und der Folgen der gesellschaftlichen Modernisierung thematisiert. ${ }^{8}$ In Deutschland gibt es jedoch nicht erst im Zuge der andauernden Finanz- und Wirtschaftskrise Indizien für eine Revitalisierung von Verteilungskonflikten, die auch mit dem Entstehen der Linkspartei parteipolitisch wirkmächtig wurden. Verteilungsfragen strukturieren die politische Auseinandersetzung und werden auch über die Richtungsbegriffe Links und Rechts ausgetragen. Vor diesem Hintergrund müssen Befunde über deren Bedeutungswandel aktualisiert werden. Hier sollen daher als zweites Forschungsziel Hypothesen zum Wandel der Bedeutungsinhalte von Links und Rechts in Deutschland innerhalb der letzten beiden Jahrzehnte getestet werden.

Auch zwanzig Jahre nach dem Mauerfall belegen zahlreiche Studien Unterschiede zwischen den Bürgern in den alten und neuen Bundesländern des wiedervereinigten Deutschlands, etwa in den Einstellungen zu politischen Institutionen oder Parteien. Nach wie vor stellt sich die Frage nach der Entwicklung einer mehr oder weniger einheitlichen Politischen Kultur. ${ }^{9}$ Wegen der unterschiedlichen Erfahrungen der ostdeutschen Bürger mit dem

5 Vgl. Dieter Fuchs / Hans-Dieter Klingemann, a.a.O. (Fn. 3), S. 204; Links-Rechts-Skala als Standardinstrument in der Wahlforschung.

6 An dieser Stelle soll nicht die Auseinandersetzung über einen Bedeutungswandel in Bezug auf die Relevanz der Links-Rechts-Dimension als politische Strukturkategorie fortgesetzt werden. Diese Diskussion wurde im Zusammenhang mit der These geführt, Links und Rechts könnten mit dem „Ende der Ideologien“ oder in wohlhabenden Gesellschaften verschwinden. Sie soll jedoch hier nicht aufgenommen werden, denn an dieser Sicht bestehen erhebliche Zweifel: Dafür dass Links und Rechts in den politischen Vorstellungswelten der Bürger nach wie vor präsent sind und nicht ein historisches Relikt aus Zeiten großer ideologischer Grabenkämpfe darstellen, spricht beispielsweise schon die verbreitete Bereitschaft und Fähigkeit der deutschen Bevölkerung, sich in Umfragen selbst auf der Links-Rechts-Dimension einzustufen. Vgl. Daniel Bell, The End of Ideology, Glencoe 1960; Tobias Dürr, Die Linke nach dem Sog der Mitte. Zu den Programmdebatten von SPD, Grünen und PDS in der Ära Schröder, in: APuZ, B21/2002, S. 5 - 12; Kai Arzheimer, Ideologien, in: Viktoria Kaina / Andrea Römmele (Hrsg.), Politische Soziologie. Ein Studienbuch, Wiesbaden 2009, S. 83 - 108, S. 100.

7 Vgl. Anthony Downs, An Economic Theory of Democracy, New York 1957.

8 Vgl. Ronald Inglehart, Traditionelle politische Trennungslinien und die Entwicklung der neuen Politik in westlichen Gesellschaften, in: PVS, 24. Jg. (1983), H. 2, S. 139 - 165.

9 Vgl. Oscar W. Gabriel, Wächst zusammen, was zusammen gehört?, in: ders. / Jürgen W. Falter / Hans Rattinger (Hrsg.), Wächst zusammen, was zusammen gehört? Stabilität und Wandel politi- 
Sozialismus sowie der westdeutschen Bevölkerung mit einer liberalen Demokratie besteht Grund zur Annahme, dass auch die Staatszielvorstellungen und Ideologien der Bürger bis heute einander nicht genau entsprechen. ${ }^{10}$ Außerdem dürften Differenzen darin bestehen, welche Bedeutungsinhalte mit Links und Rechts jeweils verknüpft werden. Mit Blick auf die oben genannten Funktionen der Richtungsbegriffe könnte dies einerseits dazu führen, dass erstens von politischen Eliten kommunizierte Inhalte in Ost und West von den Bürgern nicht in ähnlicher Weise verstanden werden und dadurch Unzufriedenheit hervorgerufen wird. Zweitens werden aufgrund der zentralen Rolle von Links und Rechts in individuellen Orientierungssystemen auch Unterschiede in anderen Dimensionen politischer Einstellungen wahrscheinlich. Andererseits ist zwei Jahrzehnte nach dem Ende der DDR aber auch denkbar, dass inzwischen einheitliche Vorstellungen von Links und Rechts in beiden Landesteilen vorherrschen. Um diese Unterschiede und Gemeinsamkeiten wird es im Folgenden gehen.

\section{Bedeutungsinhalte von Links und Rechts}

Über die Begriffe Links und Rechts werden politische Konflikte vermittelt, wahrgenommen und gedeutet. ${ }^{11}$ So beschreibt Oddbjörn Knutsen die Links-Rechts-Dimension als „overarching spatial dimension capable of incorporating many types of conflict lines"12. Die politische Ordnung selbst, aber auch die wirtschaftliche und die gesellschaftliche können Thema politischer Konflikte sein und sich im Links-Rechts-Schema manifestieren. ${ }^{13}$ So werden Streitpositionen über die wünschenswerte Politik, Gesellschaft und Wirtschaft verknüpft. Neben Werten spielen auch andere „Bedeutungsträger“, die in zentralen politischen Konflikten präsent sind, eine Rolle, nämlich soziale Gruppen, ideologische Bewegungen, politische Parteien und konkrete Issues. ${ }^{14}$ Dieter Fuchs und Hans-Dieter Klingemann gehen davon aus, dass aufgrund der vorgenommenen Bedeutungszuschreibungen zu den Symbolen Links und Rechts auf Aggregatebene wenige Kategorien einen Großteil der Assoziationen

scher Einstellungen im wiedervereinigten Deutschland, Baden-Baden 2005, S. 385 - 423, S. 385.

10 Vgl. Wolfgang Jagodzinski / Steffen M. Kühnel, Werte und Ideologien im Parteienwettbewerb, in:

Oscar W. Gabriel / Oskar Niedermayer / Richard Stöss (Hrsg), Parteiendemokratie in Deutschland, Wiesbaden 2002, S. $204-227$, S. 207.

11 Vgl. Dieter Fuchs / Steffen M. Kühnel, Die evaluative Bedeutung ideologischer Selbstidentifikation, in: Hans-Dieter Klingemann (Hrsg.), Wahlen und Wähler. Analysen aus Anlaß der Bundestagswahl 1987, Opladen 1990, S. 217 - 252, S. 220; Anja Neundorf, Growing up on Different Sides of the Wall - A Quasi Experimental Test: Applying the Left-Right Dimension to the German Mass Public, in: German Politics, 18. Jg. (2009), H. 2, S. 201 - 225, S. 203 f.

12 Oddbjörn Knutsen, Value Orientations, Political Conflicts and Left-Right Identification: A Comparative Study, in: European Journal of Political Research, 28. Jg. (1995), H. 1, S. 63 - 93, S. 87.

13 Vgl. Hans-Dieter Klingemann, Fakten oder Programmatik? Die Thesen von Murphy et al. über den Bedeutungswandel von „links“ und „rechts“ und das gegenwärtige Verständnis der politischen Richtungsbegriffe in der Bevölkerung der Bundesrepublik Deutschland, in: PVS, 23. Jg. (1982), H. 2, S. 214 - 224, S. 217; ähnlich auch Jean A. Laponce, a.a.O. (Fn. 1), S. 205.

14 Vgl. Dieter Fuchs / Hans-Dieter Klingemann, a.a.O. (Fn. 2), S. 213 ff.; Ronald Inglehart / HansDieter Klingemann, Party Identification, Ideological Preference and the Left-Right Dimension among Western Mass Publics, in: Ian Budge / Ivor Crewe / Dennis Farlie (Hrsg.), Party Identification and Beyond, New York 1976, S. 243 - 273, mit der eingeführten Unterscheidung zwischen themen- und ideologiebezogenen Interpretationen von Links und Rechts. 
abdecken und eine Art Grundkonsens in der Bevölkerung darüber vorherrscht, was mit diesen Begriffen gemeint ist. ${ }^{15}$

Zentrale Bedeutungsinhalte der Links-Rechts-Dimension stellen nach Stand der Forschung Einstellungen zum sozialen Wandel und zum Verhältnis zwischen Staat und Markt dar: „Kernelement der Bedeutung des Schemas" 16 wird die Positionierung gegenüber Veränderungen in Politik und Gesellschaft bezeichnet, so etwa bei Jean Laponce oder den Autoren um Seymour M. Lipset. ${ }^{17}$ Rechts wird dabei mit der Erhaltung des Status quo, Links mit der Befürwortung von Veränderung, insbesondere mit dem Ziel des Abbaus sozialer Ungleichheiten ${ }^{18}$ in Verbindung gebracht. Nach Anthony Downs ${ }^{19}$ geht es bei der ökonomischen Links-Rechts-Dimension, die den Wettbewerb der Parteien um Wählerstimmen entscheidend beeinflusst, um die Rolle des Staates im ökonomischen Prozess. ${ }^{20}$ Die Befürwortung von Staatseingriffen (Links) wird hier traditionell der Forderung nach unbeschränkter Marktwirtschaft, unternehmerischer Freiheit und Eigenverantwortung gegenübergestellt (Rechts).

\section{Annahmen zum Wandel der Bedeutungsinhalte von Links und Rechts in Deutschland}

Nach Norberto Bobbio gibt es so lange Polarisierung über die Links-Rechts-Dimension, wie Konflikte in Politik und Gesellschaft vorhanden sind. ${ }^{21}$ Weil sich nicht nur spezifische Konfliktkonstellationen, sondern auch deren Interpretationen ändern, ist von einem Wandel der Bedeutungsinhalte der Links-Rechts-Dimension auszugehen, zu dem in den letzten Jahrzehnten mehrere Annahmen formuliert wurden. Dabei wird dieser „im doppelten Sinne" diskutiert: im Sinne einer veränderten Relevanz von Links und Rechts als politische Strukturkategorie sowie als semantische Transformation der Links-Rechts-Dimension, auch wenn beide Aspekte des Bedeutungswandels zusammenhängen. Im Mittelpunkt dieser Analyse steht der Wandel der Bedeutungsinhalte in den Köpfen der Menschen, wobei Veränderungen hier auch Folgen für den Gebrauch der Richtungsbegriffe als Orientierungshilfe und Kommunikationsmedien zwischen Bürgern und Eliten haben. Allerdings hat sich diesbezüglich die Vermutung als überholt erwiesen, Links und Rechts würden aus dem politischen Leben verschwinden und ihre Rolle als Orientierungshilfe (zum Beispiel an andere Symbole oder Heuristiken) abgeben, weil sich die modernen Gesellschaften stark verändert haben und Verteilungskonflikte beispielsweise in wohlhabenden Ländern irrelevant geworden sind.

Was den Wandel der Bedeutungsinhalte angeht, so erscheint die als Persistenzhypothese bezeichnete Vermutung der gleichbleibenden Bedeutung der Richtungsbegriffe ${ }^{22}$ unter

15 Vgl. Dieter Fuchs / Hans-Dieter Klingemann, a.a.O. (Fn. 3), S. 206.

16 Dieter Fuchs / Steffen M. Kühnel, a.a.O. (Fn. 11), S. 221 f.

17 Vgl. Jean A. Laponce, a.a.O. (Fn. 1); Seymour M. Lipset / Paul F. Lazarsfeld / Allen H. Barton / Juan Linz, The Psychology of Voting: An Analysis of Voting Behavior, in: Lindzey Gardner (Hrsg.), Handbook of Social Psychology 2, Reading 1954, S. 1124 - 1554, S. 1135.

18 Vgl. Hans-Dieter Klingemann, a.a.O. (Fn. 13); S. 217.

19 Vgl. Anthony Downs, a.a.O. (Fn. 7).

20 Vgl. Kai Arzheimer, a.a.O. (Fn. 6), S. 98.

21 Vgl. Norberto Bobbio, Left and Right. The Significance of a Political Distinction, Chicago 1996, S. 35 .

22 Vgl. Herbert Kitschelt / Staf Hellemans, The Left-Right Semantics and the New Politics Cleavage, in: Comparative Political Studies, 23. Jg. (1990), H. 2, S. $210-238$. 
zwei Gesichtspunkten unplausibel: Sieht man Links und Rechts als von Konfliktkonstellationen in Politik und Gesellschaft abhängige Etiketten an, ist nicht zu erwarten, dass diese bei sich wandelnden Konflikten keine Bedeutungsveränderungen erfahren. Hinzu kommt, dass Links und Rechts schon öfter ihre inhaltliche Flexibilität bewiesen haben. In Abhängigkeit vom politischen und gesellschaftlichen Kontext ist seit dem Ende des 19. Jahrhunderts vor allem der sozioökonomische Konflikt zum zentralen Merkmal der Links-RechtsPolarisierung geworden. ${ }^{23}$ Doch soll sich im Zuge der gesellschaftlichen Modernisierung (unter anderem wirtschaftliche Prosperität, kognitive Mobilisierung etc.) durch die Entstehung einer neuen Spannungslinie zwischen Materialismus und Postmaterialismus ab den 1960er Jahren auch die Semantik von Rechts und Links gewandelt und sich neuen politischen Themen, Akteuren, Interessen und Zielpräferenzen angepasst haben.

Die wissenschaftliche Diskussion um den Bedeutungswandel der Links-Rechts-Dimension wurde diesbezüglich meist im Zusammenhang mit Ronald Ingleharts Theorie des Wertewandels geführt ${ }^{24}$, wobei jedoch neuere Analysen, die die Überlegungen vor dem Hintergrund aktueller politischer Kontextbedingungen prüfen können, eher selten sind. Der Transformationshypothese Ingleharts zufolge verliert der ,alte“ sozioökonomische Konflikt für die Links-Rechts-Dimension an Bedeutung und macht Auseinandersetzungen auf einer neuen Materialismus-Postmaterialismus-Konfliktachse Platz. Die politischen Richtungsbegriffe Links und Rechts könnten im Zuge dieser Entwicklung „from class-based to valuebased political polarization " 25 weniger häufig mit materiellen Interessen und verteilungspolitischen Aspekten verknüpft und stattdessen stärker von Auseinandersetzungen darüber geprägt sein, welches die wünschenswerten Ziele von Individuum, Politik und Gesellschaft sein sollen. Durch eine Veränderung der Werteprioritäten in den westlichen Ländern müssten Fragen der kulturellen Gleichheit und Entwicklung ökonomische Streitpunkte ersetzen und neue Bedeutungsinhalte im Zusammenhang mit Selbstentfaltung, Gleichstellung oder auch Schutz der Umwelt in den Mittelpunkt rücken. Eine Transformation der Begriffe soll dabei vor allem für Links und nicht mit dem früh konstatierten Wertewandelschub der 1970er Jahre, sondern später nach dem Transfer der neuen Themen von den Eliten zur Bevölkerung stattgefunden haben. ${ }^{26}$

Empirische Befunde für Deutschland sprechen nicht für einen Wegfall des „conventional economic meaning “27, zeigen aber eine Zunahme der mit der Neuen Politik verbundenen Bedeutungszuschreibungen für Links in den 1990er Jahren. ${ }^{28}$ Vor diesem Hintergrund

23 Vgl. Peter Selb, Die inhaltliche Bedeutung der Links-Rechts-Dimension 1971-1996. Invarianz oder Wandel?, Mannheim 1996 (Magisterarbeit am Lehrstuhl für Politische Wissenschaft und International Vergleichende Sozialforschung, Jan van Deth), S. 25.

24 Vgl. Ronald Inglehart, The Changing Structure of Political Cleavages in Western Society, in: Russell J. Dalton / Scott C. Flanagan / Paul A. Beck (Hrsg.), Electoral Change in Advanced Industrial Democracies: Realignment or Dealignment?, Princeton 1984, S. 25 - 69; Herbert Kitschelt I Staf Hellemans, a.a.O. (Fn. 22); Dieter Fuchs / Hans-Dieter Klingemann, a.a.O. (Fn. 3); Oddbjörn Knutsen, a.a.O. (Fn. 12).

25 Ronald Inglehart, a.a.O. (Fn. 8), S. 26.

26 Weitere Ausführungen zur sogenannten verspäteten partiellen Pluralisierung bei Peter Selb, a.a.O. (Fn. 23), S. $30 \mathrm{ff}$.

27 Herbert Kitschelt / Staf Hellemans, a.a.O. (Fn. 22), S. 214.

28 Vgl. Petra Bauer-Kaase, Politische Ideologie im Wandel? - Eine Längsschnittanalyse der Inhalte der politischen Richtungsbegriffe, links' und ,rechts', in: Hans-Dieter Klingemann / Max Kaase (Hrsg.), Wahlen und Wähler. Bundestagswahl 1998, Wiesbaden 2001, S. 207 - 243. 
ist es sinnvoll, die Überlegungen von Inglehart für eine Betrachtung Deutschlands bis zur Mitte der 1990er Jahre abzuschwächen und mit Beobachtungen über eine Pluralisierung der Bedeutungszuschreibungen zu Links und Rechts zu verbinden. Dazu lautet die Hypothese H1a: In einer Phase zumindest bis Mitte der 1990er Jahre kam es zu einer Zunahme von Aspekten Neuer Politik im semantischen Raum von Links und zu einem Rückgang von mit Verteilungsaspekten verbundenen Bedeutungsinhalten. In Anlehnung an Odbjörn Knutsen ${ }^{29}$ wird davon ausgegangen, dass die linke Seite der Dimension um Neue Politik ergänzt wird, ohne dass jedoch linksmaterialistische Fragen völlig verdrängt werden, auch wenn diese weniger häufig mit Links und Rechts assoziiert werden (im Ergebnis also eine Pluralisierung der Bedeutungszuschreibungen).

Dass sich dieser Trend allerdings bis ins neue Jahrtausend hinein fortgesetzt hat, ist anzuzweifeln. Vieles spricht dafür, dass im Laufe der 1990er Jahre die Herausforderungen für den Sozialstaat wie der demographische Wandel, finanzielle Lasten der Wiedervereinigung und Probleme am Arbeitsmarkt zu einer Revitalisierung ökonomischer Themenkomplexe und Fragen nach einer angemessenen Rolle des Staates und einer gerechten Gesellschaft geführt haben. ${ }^{30}$ Zum einen lässt sich seit Mitte der 1990er Jahre eine verstärkte Wahrnehmung und Thematisierung ungleicher Verteilung in der Öffentlichkeit beobachten. ${ }^{31}$ Zum anderen hat sich der neu aufkommende Sozialstaatskonflikt auch im bundesdeutschen Parteienwettbewerb niedergeschlagen ${ }^{32}$, wobei sich die Linkspartei im Zuge der Debatten um die Agenda 2010 als "gesamtdeutsche Linksalternative zur SPD“ positioniert hat. ${ }^{33}$ Aber auch in den Wahlkämpfen und bei der Stimmabgabe im Zuge der Bundestagswahlen seit 1998 spielte der Themenbereich Sozialstaat/soziale Gerechtigkeit eine zentrale Rolle. Die Anpassung des Schemas wird wahrscheinlich zügig stattgefunden haben, wenn man bedenkt, dass Fragen der Gerechtigkeit sehr schnell aus Teilen der Eliten heraus thematisiert und in Wahlkämpfen durch alte und neue Parteien ständig aktualisiert wurden. In Anbetracht der skizzierten Veränderungen gesellschaftlicher Konflikte und ihrer Interpretationen

29 Vgl. Oddbjörn Knutsen, a.a.O. (Fn. 12).

30 Der Argumentation von Markus Klein und Dieter Ohr zur Richtung des Wertewandels folgend, könnte man ergänzen, dass trotz eines relativ hohen Lebensstandards in Deutschland materielle Fragen bei einer Verschlechterung der wirtschaftlichen Situation in den 1990er Jahren wieder eine besondere Rolle spielen, weil sich Bürger aufgrund eines relativ hohen Anspruchsniveaus leicht depriviert fühlen. Vgl. Markus Klein / Dieter Ohr, Ändert der Wertewandel seine Richtung? Die Entwicklung gesellschaftlicher Wertorientierungen in der Bundesrepublik Deutschland zwischen 1980 und 2000, in: Rüdiger Schmitt-Beck / Martina Wasmer / Achim Koch (Hrsg.), Sozialer und politischer Wandel in der Bundesrepublik Deutschland. Analysen mit Allbus-Daten aus zwei Jahrzehnten, Opladen 2004, S. 153 - 178, S. 157.

31 Bernhard Weßels spricht davon, dass in den 1990er Jahren von den Bürgern zunehmend eine Gerechtigkeitslücke wahrgenommen wurde. Ulrich Eith und Gerd Mielke beobachten eine neue Bedeutung der sozialen Frage. Vgl. Bernhard Weßels, Kanzler- oder Politikwechsel? Bestimmungsgründe des Wahlerfolgs der SPD bei der Bundestagswahl 1998, in: Jan van Deth / Hans Rattinger / Edeltraud Roller (Hrsg.), Die Republik auf dem Weg zur Normalität?, Opladen 2000, S. 35 65, S. 63; Ulrich Eith / Gerd Mielke, Die soziale Frage als „neue“ Konfliktlinie? Einstellungen zum Wohlfahrtsstaat und zur sozialen Gerechtigkeit und Wahlverhalten bei der Bundestagswahl 1998, in: Jan van Deth / Hans Rattinger / Edeltraud Roller (Hrsg.), a.a.O. (Fn. 31), S. 93 - 115.

32 Vgl. Oskar Niedermayer, Die Entwicklung des Bundesdeutschen Parteiensystems, in: Frank Decker / Viola Neu (Hrsg.), Handbuch der deutschen Parteien, Wiesbaden 2007, S. 114 - 135, S. $128 \mathrm{ff}$.

33 Vgl. Tobias Dürr, a.a.O. (Fn. 6). 
ist für eine zweite Phase ab Mitte der 1990er Jahre sowohl in West- als auch in Ostdeutschland mit einer stärkeren Präsenz von mit Gerechtigkeit und sozialer Sicherheit verbundenen Assoziationen und - in einer Fortführung der Überlegungen zur vorigen Hypothese mit einem Rückgang von Aspekten Neuer Politik (für Links) zu rechnen (H1b).

Vorhandene theoretische Überlegungen zum Bedeutungswandel des Schemas entstammen vor allem den Diskussionen um alte materialistische Fragen sowie Neue Politik und enthalten größtenteils nur Szenarien für veränderte Bedeutungszuschreibungen zu Links; ein möglicher Bedeutungswandel auf der rechten Seite des Spektrums wird in diesem Rahmen nicht diskutiert. Bei den Diskussionen um einen Wertewandel in westlichen Gesellschaften wird allerdings vereinzelt auch auf einen Bedeutungswandel rechter Positionen verwiesen. ${ }^{34}$ Im Zuge des Aufkommens der „New Right“ müssten Autorität, Sicherheit und Tradition stärker thematisiert werden als materialistische Fragen. In Anlehnung an diese Überlegungen und parallel zu den Annahmen über den Bedeutungswandel von Links erwarten wir zunächst eine Abnahme von Nennungen im Zusammenhang mit rechtsmaterialistischen beziehungsweise wirtschaftlichen Themen und eine Zunahme von Aspekten Neuer Politik, die stärker Fragen der Gesellschaftsordnung betreffen (H1c). ${ }^{35}$ Analog zu Links wäre dann ab Mitte der 1990er Jahre wieder ein umgekehrter Trend zu erwarten - mit einer Zunahme von Nennungen zum Thema Marktwirtschaft, Eigenverantwortung etc. (H1d).

Für die Zeit nach der Wiedervereinigung rücken außerdem Bedeutungsverschiebungen auf einer anderen Ebene in den Mittelpunkt: Der Mauerfall vor zwanzig Jahren steht auch für einen Wegfall von Systemalternativen. Themen im Zusammenhang mit Kommunismus und Sozialismus, deren Realisierung in den Ländern des damaligen Ostblocks gescheitert ist, dürften seither an Wichtigkeit in Gesamtdeutschland verloren haben und sich auch in den Bedeutungszuschreibungen für das Etikett Links niederschlagen. Weil keine Gegenentwürfe für Politik, Gesellschaft und Wirtschaft im Raum stehen, kann man davon ausgehen, dass auch die eigene Ordnung in Deutschland weniger diskutiert wird. Parallel zu den Veränderungen auf der linken Seite des ideologischen Spektrums müsste man daher auch für Rechts einen Rückgang von mit der kapitalistischen Ordnung verbundenen Nennungen beobachten können (H1e).

\section{Links und Rechts im wiedervereinigten Deutschland}

\subsection{Annahmen für eine innerdeutsche Analyse der Bedeutungsinhalte}

Für Deutschland ergibt sich zwanzig Jahre nach dem Mauerfall eine besondere Sachlage. Kurz gesagt geht es dabei um die Gemeinsamkeiten oder Unterschiede im heutigen Begriffsverständnis zwischen Ost und West vor dem Hintergrund der geteilten Geschichte beider Landesteile.

Unterschiedliche Ausprägungen zwischen Ost- und Westdeutschen in ihren ideologischen Positionierungen sind bekannt. So stufen sich in Ostdeutschland mehr Personen links der Mitte ein als im westlichen Teil des Landes. ${ }^{36}$ Möglicherweise unterscheiden sich

34 Vgl. Scott C. Flanagan, Value Change in Industrial Societies, in: American Political Science Review, 81. Jg. (1987), H. 4, $1303-1319$.

35 Vgl. ebenda, S. 1306.

36 Vgl. Kai Arzheimer, a.a.O. (Fn. 6), S. 100. 
die Bürger in den alten und neuen Bundesländern auch in ihrem Verständnis der Richtungsbegriffe. Unterschiede wären mit Sozialisation oder Situation begründbar: Das Begriffsverständnis von Links und Rechts entwickelt sich abhängig von Politik und Gesellschaft in einem Land - Peter Mohler spricht von einer „kulturspezifische(n) Konfliktlinie,

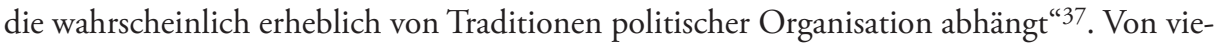
len Forschern wird die Auffassung geteilt, dass ungefähr die ersten zwanzig Lebensjahre eines Individuums entscheidend sind für die Herausbildung seiner politischen Ansichten und dass die Erfahrungen aus dieser Zeit seine politischen Einstellungen, wie etwa die Interpretationen von Links und Rechts, formen. Demzufolge kann man davon ausgehen, dass sich die Befragten in Ost und West größtenteils dahingehend unterscheiden, dass sie die für sie prägenden Erfahrungen in zwei unterschiedlichen politischen Systemen gemacht haben. Weil die Konfliktkonstellationen und deren Interpretation im autokratischen System der DDR mit einer sozialistischen Planwirtschaft und dem herrschenden Diskurs der Sozialistischen Einheitspartei andere waren als im demokratischen System der Bundesrepublik mit sozialer Marktwirtschaft und dem Wettbewerb von Parteien unterschiedlicher ideologischer Richtungen, können auch heute noch Unterschiede in den Assoziationen für Links und Rechts in den beiden Landesteilen erwartet werden.

Ein anderer Grund für solche Unterschiede könnte darin liegen, dass die Lebensverhältnisse der Bürger und einzelne Bereiche des politischen Wettbewerbs, insbesondere der Parteienwettbewerb ${ }^{38}$, in Ost und West nicht deckungsgleich sind. Folgt man den obigen Überlegungen zur frühen politischen Sozialisation, dürfte dies vor allem für die jüngere Bevölkerung zutreffen. Wenn man jedoch zusätzlich davon ausgeht, dass die mit Links und Rechts verbundenen Assoziationen der Bürger auch kurzfristig wandelbar sind (Situationsargument), kann dies für die gesamte Bevölkerung zutreffen.

Vor dem Hintergrund dieser Argumentation lassen sich konkrete Erwartungen zu den heute in Ost und West genannten Begriffsinhalten von Links und von Rechts formulieren: Für den linken Pol des Schemas kann man Unterschiede bei Nennungen mit Bezug zu Terminologien des klassischen Sozialismus erwarten. Auf das Links-Rechts-Verständnis der in der ehemaligen DDR sozialisierten Befragten wirken möglicherweise immer noch die im SED-Staat propagierten Klassenkampf-Ideologien nach. ${ }^{39}$ Dies lässt für Links eine häufigere Nennung der Ideologien Kapitalismus, Sozialismus, Kommunismus etc. erwarten (H2a).

Fragen der sozialen Sicherheit und der Gerechtigkeit können vor dem Hintergrund der in der DDR stark präsenten Diskurse um Gleichheit und Sicherheit der Bürger als zentrale Charakteristika des eigenen Systems ebenfalls bei den Assoziationen ostdeutscher Bürger zu Links häufiger auftreten (H2b). ${ }^{40}$ Ungleiche Verteilungen der mit Gerechtigkeit verbundenen Nennungen sind auch deswegen zu erwarten, weil materielle Fragen im östlichen Landesteil aufgrund der insgesamt schlechteren wirtschaftlichen Bedingungen nach wie vor stärker im Vordergrund stehen. Weil die Bedeutungsinhalte der ideologischen Etiketten

37 Peter Mohler, Links oder Rechts - Eine offene Frage, in: Cornelia Züll / Peter Mohler (Hrsg.), Textanalyse - Anwendungen der computerunterstützten Inhaltsanalyse. Beiträge zur 1. TEXTPACK-Anwenderkonferenz, Wiesbaden 1993, S. 213 - 224, S. 214.

38 Vgl. Elmar Wiesendahl, Parteien, Frankfurt am Main 2006.

39 Vgl. Klaus Schroeder, Der SED-Staat. Partei, Staat und Gesellschaft 1949-1990, München / Wien 1998.

40 Vgl. Manfred G. Schmidt, Sozialpolitik der DDR, Wiesbaden 2004. 
davon beeinflusst werden, welche Positionen die politischen Eliten, vor allem die Parteien, in ihren Diskursen vermitteln und mit diesen Etiketten verbinden, kann sich das größere Gewicht der Linkspartei im Ostteil der Republik auch auf die Bedeutungszuschreibungen in der Bevölkerung auswirken: Als Folge ist ebenfalls eine stärkere Thematisierung von Verteilungsfragen in der Bevölkerung zu vermuten. ${ }^{41}$ Auch die in Ostdeutschland heute lautere Forderung nach mehr sozialer Gerechtigkeit und mehr Staatstätigkeit wird sich möglicherweise in einer größeren Zahl solcher Bedeutungszuschreibungen für Links und Rechts ausdrücken. ${ }^{42}$

Für den Begriff Rechts könnten „einem sozialistischen Begriffsverständnis entsprechend wohl häufiger Nennungen wie Faschismus und Extremismus assoziiert “43 ${ }^{\text {werden }}{ }^{44}$, denn der Antifaschismus stellte eine zentrale Legitimationsideologie der DDR dar. ${ }^{45}$ Nicht nur die unterschiedliche Vergangenheit der beiden Landesteile, sondern auch Merkmale des Parteienwettbewerbs seit den 1990er Jahren können dafür verantwortlich sein. Zwar kommen rechtsextreme Parteien in der Bundesrepublik nach wie vor auf geringe Stimmenanteile, doch waren / sind sie speziell in einigen ostdeutschen Parlamenten auf Landes- oder Gemeindeebene präsent. Folglich kann man davon ausgehen, dass in den neuen Bundesländern die Befragten tendenziell häufiger mit Faschismus und Extremismus verbundene Interpretationen von Rechts liefern als in den alten Bundesländern (H2c).

Assoziationen mit Bezug zur kapitalistischen Wirtschaftsordnung oder zu konservativen oder traditionellen Aspekten einer Gesellschaft hingegen treten in Ostdeutschland womöglich spärlicher auf als in Westdeutschland (H2d). Dafür spricht, dass in den alten Bundesländern über einen längeren Zeitraum hinweg, insbesondere von Mitte-Rechts-Parteien, Begriffe wie konservativ, stabil, christdemokratisch, Kapitalismus, Marktwirtschaft oder Eigenverantwortung thematisiert wurden. ${ }^{46}$

Zwanzig Jahre nach dem Mauerfall sprechen jedoch auch gewichtige Gründe dafür, dass sich Ost- und Westdeutsche im Verständnis von Links und Rechts im Großen und Ganzen ähnlich sind. Zum einen herrscht in der sozialwissenschaftlichen Forschung Uneinigkeit darüber, in welchem Ausmaß die Sozialisation im autoritären SED-Regime die politischen Einstellungen der früheren DDR-Bürger tatsächlich geprägt hat. ${ }^{47} \mathrm{Zum}$ anderen stellt sich die Frage, ob diese vergangenen Erfahrungen für heutige Konfliktdeutungen und Vorstellungen von Links und Rechts relevant sind, oder ob nicht eher die Tatsache, dass die Bevölkerung schon zwanzig Jahre im selben politischen System lebt, ausschlaggebend ist. Dieses System ist für alle schon lange der übergeordnete Rahmen, in dem politische Konflikte vermittelt

41 Vgl. Susumu Shikano / Franz Urban Pappi, a.a.O. (Fn. 4), S. 20.

42 Diese Verbindung zwischen kognitivem Verständnis der untersuchten Symbole und ihrer evaluativen Bedeutung wurde schon von Pamela Conover und Stanley Feldman herausgestrichen. Vgl. Pamela J. Conover / Stanley Feldman, The Origins and Meaning of Liberal/Conservative Self-Identifications, in: American Journal of Political Science, 25. Jg. (1981), H. 4, S. 617 - 645, S. 621.

43 Peter Selb, a.a.O. (Fn. 23), S. 81.

44 Vgl. auch Anja Neundorf, a.a.O. (Fn. 11).

45 Vgl. Herfried Münkler, Antifaschismus und antifaschistischer Widerstand als politischer Gründungsmythos der DDR, in: APuZ, B45/1998, S. 16 - 29; Werner Müller, Die DDR in der deutschen Geschichte, in: APuZ, B28/2001, S. $43-53$.

46 Vgl. Wolfgang Jagodzinski / Steffen M. Kühnel, a.a.O. (Fn. 10).

47 Vgl. Russell J. Dalton, Communists and Democrats: Democratic Attitudes in the Two Germanies, in: British Journal of Political Science, 24. Jg. (1994), H. 4, S. $469-493$. 
und mithilfe des Links-Rechts-Schemas strukturiert werden, auch wenn sich die wirtschaftlichen Bedingungen unterscheiden. Auch die Parteien als wichtige Verfechter der Konflikte um Inhalte und Deutungen von Links und Rechts verstehen sich als bundesweite Akteure.

Dieser Argumentation folgend, die den Stellenwert der DDR-Sozialisation beschränkt und vor allem die Relevanz des gemeinsamen politischen Systems im wiedervereinigten Deutschland heraushebt ${ }^{48}$, soll eine konkurrierende Hypothese formuliert werden, wonach Gemeinsamkeiten im Begriffsverständnis von Links und Rechts zwischen Ost- und Westdeutschen angenommen werden ( $\mathrm{H} 2 \mathrm{e})$.

\subsection{Annahmen zur innerdeutschen Dynamik im Begriffsverständnis von Links und Rechts}

Die Gründe, die für die in den Hypothesen 1a bis 1c erwarteten Veränderungen der Begriffsinhalte verantwortlich sein sollen, gelten in den alten und neuen Landesteilen höchstwahrscheinlich nicht in gleichem Maße. Daher wird bei den Befunden in Bezug auf diese Hypothesen auch zwischen Ost und West zu unterscheiden sein. Im Rahmen dieses Vergleichs ist aber auch zu diskutieren, welche innerdeutsche Dynamik zur aktuellen Wahrnehmung der beiden Richtungsbegriffe bei Ost- und Westdeutschen geführt hat. Verschiedene Verläufe sind denkbar (vgl. Abbildung 1): Heutige Bedeutungszuschreibungen können das Resultat einer Auseinanderentwicklung, einer Annäherung oder einer seit der Wiedervereinigung parallel laufenden Entwicklung darstellen, wobei Links und Rechts nicht derselben Dynamik unterliegen müssen.

\begin{tabular}{|c|c|c|c|}
\hline \multicolumn{4}{|c|}{ Abbildung 1: Die innerdeutsche Dynamik im Begriffsverständnis von Links und Rechts } \\
\hline & & \multicolumn{2}{|c|}{ Bedeutungsinhalte heute } \\
\hline & & ähnlich & unterschiedlich \\
\hline \multirow{2}{*}{$\begin{array}{l}\text { Wandel im Verhältnis } \\
\text { Ost-West }\end{array}$} & ja & Annäherung & Auseinanderentwicklung \\
\hline & nein & Parallel Gemeinsamkeiten & Parallel Unterschiede \\
\hline
\end{tabular}

Für die Annahme, dass sich die Assoziationen von Ost- und Westdeutschen angenähert haben, sprechen - wenn man innerhalb des Sozialisationstheorems argumentiert - vor allem nachwachsende Kohorten: Für immer mehr Jahrgänge findet die prägende Vermittlung politischer Einstellungen im vereinigten Deutschland statt. Im Unterschied dazu wird die Zahl der Bürger, die den überwiegenden Teil ihres Lebens in der DDR beziehungsweise in der alten Bundesrepublik verbracht haben, immer kleiner. Je länger dabei die Periode ist, in der innerhalb eines einzigen politischen Systems Konflikte thematisiert und ausgetragen werden, desto größer dürften die Ähnlichkeiten im Verständnis von Links und Rechts bei den Bürgern in den alten und neuen Bundesländern werden (Annäherung, H3a).

Allerdings ist bei Ähnlichkeiten im Verständnis eine Annäherung dann weniger wahrscheinlich als eine parallele Entwicklung, wenn sich Ost- und Westdeutsche schon zum

48 Vgl. Katja Neller, Getrennt vereint? Ost-West-Identitäten, Stereotypen und Fremdheitsgefühle nach 15 Jahren deutscher Einheit, in: Jürgen W. Falter / Oscar W. Gabriel / Hans Rattinger / Harald Schoen (Hrsg.), Sind wir ein Volk? Ost- und Westdeutschland im Vergleich, München 2006, S. 13 - 36, S. 17 ff. 
Zeitpunkt der Wende in ihren Vorstellungen von Links und Rechts ähnlich waren. Empirische Anhaltspunkte für einige Gemeinsamkeiten in den ersten Jahren nach der Wiedervereinigung gibt es. ${ }^{49}$ Vor allem sprechen auch Erkenntnisse aus der Forschung zur politischen Unterstützung der ostdeutschen Bürger direkt nach der Wende für die Möglichkeit, dass es den Autoritäten im SED-Staat nicht gelungen ist, die Normen und Konfliktdeutungen des Regimes in der Bevölkerung zu verankern. So ergaben die ersten Meinungsumfragen in der Zeit nach der Wiedervereinigung, dass demokratische Einstellungen von Ostdeutschen mindestens in gleichem Ausmaße geäußert wurden wie von westdeutschen Befragten, was mit Erstaunen zur Kenntnis genommen wurde. ${ }^{50}$ Gründe hierfür können auch Ähnlichkeiten der Interpretationen von Links und Rechts durch die Bevölkerung in beiden Landesteilen Anfang der 1990er Jahre bedingt haben (H3b): Einige Autoren gehen davon aus, dass es in der DDR einen Kontrast zwischen sozialistischer Propaganda und dem politischen Alltag gab, und die Bürger daher früh mit einer gewissen Distanz zu den Konfliktdeutungen des Sozialismus aufwuchsen. ${ }^{51}$ Hinzu kam der Einfluss einer „,counter-cultural socialization “ insofern, als die Durchlässigkeit der innerdeutschen Grenze über Medien oder Familienbindungen gegenläufige Vorstellungen zugänglich machte. ${ }^{52}$

Dass Unterschiede im Verständnis von Links und Rechts über die letzten zwanzig Jahre nach dem Mauerfall anhalten, erscheint sowohl aus der Situations- als auch aus der Sozialisationsperspektive plausibel ( $\mathrm{H} 3 \mathrm{c})$. Wenn der Rahmen, in dem Deutungen politischer Konflikte durch Eliten und Bürger stattfinden, über die Jahre nach der Wiedervereinigung bei Ost- und Westdeutschen nicht derselbe war oder nicht in gleicher Weise erlebt wurde, dann dürften die mit Links und Rechts verbundenen Assoziationen der Bürger in beiden Landesteilen über die Zeit hinweg Differenzen aufweisen.

Als ein viertes Szenario innerdeutscher Dynamik können sich die Assoziationen auch auseinanderentwickelt haben (H3d). So ist einerseits denkbar, dass sich Unterschiede in der Wahrnehmung der Lebenssituation durch ostdeutsche Bürger heute stärker im Verständnis des Links-Rechts-Schemas niederschlagen, weil es sich inzwischen als Schema zur Deutung politischer Konflikte bewährt hat. Andererseits werden möglicherweise die Deutungsangebote der Linkspartei heute mit zunehmendem Abstand zur SED-Vergangenheit eher akzeptiert.

\section{Daten und Messung}

Offene Fragen zum Bedeutungsgehalt des Links-Rechts-Schemas gehören, anders als die Links-Rechts-Selbsteinstufung, nicht zum Standardrepertoire sozialwissenschaftlicher Umfragen. Insbesondere für die Längsschnittuntersuchung des Bedeutungswandels stellt sich die Datenlage als schwierig dar, da für die gesamte Zeitreihe vergleichbare Kodierverfahren

49 Vgl. Petra Bauer-Kaase, a.a.O. (Fn. 28).

50 Vgl. ebenda.

51 Vgl. ebenda, S. 474.

52 Russell Dalton spricht von „dissonant political cues that had undermined the norms of the regime.“ Russell J. Dalton, a.a.O. (Fn. 47), S. 481. Vgl. Katja Neller, DDR-Nostalgie. Dimensionen der Orientierungen der Ostdeutschen gegenüber der ehemaligen DDR, ihre Ursachen und politischen Konnotationen, Wiesbaden 2006, S. 68. 
nötig sind. ${ }^{53}$ Neben einer eigenen Erhebung im Rahmen des von der DFG geförderten Projektes „Der Einfluss des politischen Vertrauens auf die Unterstützung der Reform des Sozialstaates in Deutschland“" (Projektnummer 6A424/6-1) an der Universität Stuttgart beziehen wir uns für die Längsschnittanalyse auf zwei frühere Studien.

Für den Erhebungszeitpunkt 1991 stützen wir uns auf eine 1996 in Mannheim angefertigte Magisterarbeit von Peter Selb, die auf eine Umfrage der Konrad-Adenauer-Stiftung (KAS-Frühjahrsstudien) zurückgreift. In repräsentativen Umfragen wurde 3.053 Bürgern in Ost und West die Frage gestellt: „Wenn in der Politik die Begriffe ,rechts' und ,links' verwendet werden, was verstehen Sie dann darunter? Was verstehen Sie unter Rechts? Und noch etwas, was Ihnen dazu einfällt?" 54

Die Daten der Erhebungszeitpunkte 1997/1998 stammen aus einer Arbeit von Petra Bauer-Kaase ${ }^{55}$, wobei die Autorin die Zeitreihe von $S_{e l b}{ }^{56}$ mit den nachfolgend beschriebenen Daten fortführt. 1997 wurden von GFM-Getas mit dem SowiBus III/1997 2.143 westdeutsche und 1.102 ostdeutsche Bürger befragt. 1998 waren es im Rahmen des SowiBus II/1998 in Westdeutschland 1.000 und in Ostdeutschland 1.007 Personen. Die Daten von 1997 und 1998 wurden gepoolt, um größere Fallzahlen zu erhalten. ${ }^{57}$ Der Fragewortlaut war bei beiden Erhebungen derselbe: „Können Sie mir bitte nun noch sagen, was Sie persönlich unter den Begriffen Links und Rechts verstehen, wenn es um Politik geht? Und was wäre sonst noch dazu zu sagen? Und noch etwas, fällt Ihnen dazu noch etwas ein?"

Für die Daten von 2007 wurden von Infratest-Dimap 1.102 Personen in Westdeutschland und 712 Personen in Ostdeutschland befragt. Nach ihrer Einstufung auf der LinksRechts-Skala wurden die Befragten gebeten zu definieren, was sie unter Links und Rechts verstehen: „In der Politik spricht man manchmal von ,links' und ,rechts'. Wo auf der Skala würden Sie sich selbst einstufen, wenn 1 für links steht und 11 für rechts?"; und „Was bedeutet für Sie ,Links' in der Politik? Was bedeutet für Sie ,Rechts’ in der Politik?“. Bei allen Studien wurde die Links-Rechts-Selbsteinstufung vor der Erhebung der offenen Nennungen abgefragt.

Die inhaltsanalytische Auswertung der offenen Fragen erfolgte mittels eines Codeschemas. Um die bestehende Zeitreihe fortsetzen zu können, wurde auch für die aktuellen Nennungen von 2007 das Codeschema von Selb verwendet. ${ }^{58}$ Dieser nutzte für seine Studien die in den Datensätzen der KAS bereits vorhandenen Kodierungen und vereinheitlichte diese zu 57 Kategorien für die Bedeutung von Rechts und 64 Kategorien für Links. In einem weiteren Schritt wurden sie dann jeweils nach inhaltlicher Ähnlichkeit zu übergeordneten Be-

53 Vgl. Peter Selb, a.a.O. (Fn. 23), S. 45; Petra Bauer-Kaase, a.a.O. (Fn. 28), S. 214.

54 Peter Selb, a.a.O. (Fn. 23).

55 Vgl. Petra Bauer-Kaase, a.a.O. (Fn. 28).

56 Vgl. Peter Selb, a.a.O. (Fn. 23).

57 Vgl. Petra Bauer-Kaase, a.a.O. (Fn. 28), S. 215.

58 Zwar gibt es auch von Dieter Fuchs und Hans-Dieter Klingemann ein bewährtes Codeschema; doch aus zwei Gründen greifen wir auf die gut dokumentierte Arbeit von Peter Selb zurück. Zum einen stehen uns für das erstgenannte Codeschema zwar die Daten, nicht jedoch detaillierte Kodieranweisungen zur Verfügung, zum anderen eignet sich das stärker an den damaligen Nennungen orientierte Schema von Peter Selb besser zur Überprüfung des Wandels konkreter Inhalte anstatt abstrakterer Kategorien. Vgl. Dieter Fuchs / Hans-Dieter Klingemann, a.a.O. (Fn. 3); Peter Selb, a.a.O. (Fn. 23). 
griffskategorien zusammengefasst. ${ }^{59}$ Auch Petra Bauer-Kaase verwendet dieses Schema. Bei der Kodierung der Nennungen von 2007 wurde es in seinen Unterkategorien etwas erweitert, aber es wurde darauf geachtet, dass der vorgegebene Rahmen der übergeordneten Begriffskategorien, die nachfolgend analysiert werden, beibehalten wurde. Somit kann die Vergleichbarkeit des Codeschemas gewährleistet werden. ${ }^{60}$ Das minimal modifizierte Schema stellt nun für Rechts 58 und für Links 69 Unterkategorien bereit, in die die offenen Nennungen kodiert werden können. ${ }^{61}$ Diese lassen sich nach $S_{e l b}{ }^{62}$ in 14 beziehungsweise 17 übergeordnete Kategorien integrieren.

\section{Aktuelle Ergebnisse zum Bedeutungsgehalt und Bedeutungswandel der politischen Begriffe Links und Rechts}

Bereits vor dem Vergleich der inhaltlichen Dimensionen der Begriffe Links und Rechts lassen sich zwischen den beiden Landesteilen in Bezug auf die Nennungsbereitschaft deutliche Unterschiede feststellen. So sind in Ostdeutschland nur 25 Prozent der 712 Befragten nicht in der Lage oder bereit sich zu äußern, wobei kein Unterschied bezüglich der Richtung der Begriffe festzustellen ist (vgl. Tabelle 1 und 2). In Westdeutschland fallen dagegen 36 Prozent der 1102 Befragten bei Links als auch bei Rechts in diese Kategorie. Des Weiteren gaben ostdeutsche Befragte, die die beiden Begriffe definieren konnten - relativ mehr Nennungen ab als Westdeutsche, die von dieser Möglichkeit weniger Gebrauch machten. Diesen Zahlen zufolge kann man nicht von einer Bedeutungsarmut der Links-Rechts-Dimension in den neuen Bundesländern sprechen, die durchaus nach mehreren Jahrzehnten in einem System ohne freien Wettbewerb von Konfliktparteien denkbar gewesen wäre. Aus den kodierten Nennungen lassen sich mehrere übergeordnete Bedeutungskategorien identifizieren, anhand derer zunächst aktuelle Unterschiede und Gemeinsamkeiten zwischen Ost- und Westdeutschland herausgearbeitet werden können, bevor Links und Rechts im Hinblick auf den inhaltlichen Bedeutungswandel untersucht werden. ${ }^{63}$

\subsection{Der Bedeutungsgehalt des politischen Richtungsbegriffes Rechts in Ost- und Westdeutschland}

Für den politischen Richtungsbegriff Rechts lassen sich anhand der Daten für das Jahr 2007 in Ost- und Westdeutschland mehrere übergeordnete Begriffskategorien identifizieren, die einen Grundkonsens über seine Inhalte zwanzig Jahre nach dem Mauerfall wider-

59 Vgl. Peter Selb, a.a.O. (Fn. 23), S. 49.

60 Wir sind uns bewusst, dass in manchen Fällen (zum Beispiel Kategorie 128 Links - Familie, 22 Links - KPD/DKP, 34 Rechts - Studenten) die Zuordnung unter eine übergeordnete Begriffsklasse zweifelhaft ist oder nach anderen Gesichtspunkten hätte erfolgen können. Um die Vergleichbarkeit zu den Daten der früheren Studien zu gewährleisten, wurde davon abgesehen Unterkategorien herauszulösen und andernorts einzugliedern, zumal diese Nennungen nur einen marginalen Anteil aufweisen.

61 Das vollständige Codeschema kann abgerufen werden unter http://www.uni-stuttgart.de/soz/ avps/mitarbeiter/Online-Anhang_Truedinger_Bollow_2011.pdf.

62 Vgl. Peter Selb, a.a.O. (Fn. 23).

$63 \mathrm{Da}$ es sich bei der Zeitreihe nicht um Paneldaten handelt, können wir auf der Individualebene keine Aussagen darüber treffen, ob sich die Vorstellungen der einzelnen Bürger geändert haben. 
spiegeln. Entsprechend der formulierten Hypothesen sind jedoch auch strukturelle Unterschiede in den mit Rechts verbundenen Inhalten zwischen den alten und neuen Bundesländern zu vermuten.

Der Bedeutungskern von Rechts wird von Assoziationen zu „Extremismus“ und „traditionellen Mitte/Rechts-Parteien, Organisationen und Eliten" bestimmt, die über ein Drittel aller Nennungen ausmachen. Insbesondere in letztgenannter Kategorie sowie der Dimension der „traditionellen Gesellschaftsordnung“, worunter etwa Aussagen zu Konservatismus fallen, zeigt sich die der rechten Seite des Schemas zugeschriebene Status-quo Orientierung. Weitere wichtige Inhalte gehören zur Gruppe der „ideologischen Bewegungen“ und enthalten Antworten mit Bezug zu Nationalsozialismus und Faschismus oder sind Äußerungen zur „kapitalistischen Wirtschaftsordnung“. Nennungen zum Beispiel von „gesellschaftlichen Gruppen“ oder von „Patriotismus“, wie auch die übrigen im Codeschema enthaltenen Kategorien, sind für diese Seite des Schemas nur von untergeordneter Bedeutung. „Idiosynkratische Bezüge" und "Sonstiges", worunter auch falsche Nennungen eingeordnet sind, fallen zwar ins Gewicht, bilden aber keine inhaltlich interpretierbare Bedeutung für das Schema.

Nennungen, die „ideologische Bewegungen“ betreffen, sollten den obigen Überlegungen zufolge in den neuen Bundesländern häufiger anzutreffen sein, da die politische Sozialisation in der DDR besonders von der ideologischen Abgrenzung zum „faschistischen Ausland“ geprägt war. Diese Vermutung bestätigt sich. So verbinden Befragte aus den östlichen Bundesländern mit zehn Prozent der Nennungen doppelt so häufig Assoziationen zu „ideologischen Bewegungen" wie Nationalsozialismus und Faschismus mit Rechts (vgl. Tabelle 1). Zusammen mit der Bedeutungskategorie „Diktatur “64, die acht Prozent der Nennungen in den neuen Bundesländern ausmacht, zeigt sich deutlich, dass die Abgrenzungsideologie noch in den Köpfen der Menschen fortwirkt. Außerdem schlägt die unterschiedliche Situation in den beiden Landesteilen zu Buche: Nicht nur ein am rechten Rand erweitertes Parteienspektrum und die höhere Wahrscheinlichkeit eines direkten Kontakts mit dem Phänomen in Folge einer größeren Verbreitung rechtsextremer Gruppen können Erklärungen dafür liefern, dass in den neuen Bundesländern der Begriff Rechts stärker mit Erwähnungen von Rechtsextremismus etc. einhergeht (H2c). Die Hypothese wird von den Daten gestützt: Nennungen, die auf „Extremismus“ verweisen, bilden mit 30 Prozent in Ostdeutschland und 20 Prozent in Westdeutschland in die wichtigste Bedeutungskategorie von Rechts. Die hohen Werte in Ost und West zeigen aber auch, dass das Thema im ganzen Land in den Medien und der Politik präsent ist, wie nicht zuletzt an immer wieder aufkommenden Forderungen nach Parteiverboten deutlich wird. Rechtsextremismus wird als gesamtgesellschaftliches Problem wahrgenommen, das nicht nur für die Ostdeutschen im Rahmen des Schemas Relevanz besitzt.

64 Bemerkenswert ist der Wert von acht Prozent der Nennungen für die Kategorie „Diktatur“ in Ostdeutschland, der nicht zu den früheren Befunden sowie den Werten in Westdeutschland passt. Dieser ist wohl auf eine Eigenheit des Codeschemas zurückzuführen, das abstrakten rechten ideologischen Bewegungen eine übergeordnete Kategorie zuweist und Assoziationen zur konkreten Umsetzungen zum Beispiel im Dritten Reich als „Diktatur“ eine weitere Kategorie zur Verfügung stellt. Da keine genauen Anweisungen der früheren Codierungen vorliegen, sind hier Abweichungen zwischen den Datensätzen bei der Codierung der Kategorie Diktatur möglich. Dennoch ist der Wert substantiell zu interpretieren, da - egal welcher der beiden Kategorien man die Nennungen zurechnet - ein auffallend hoher Wert zu verzeichnen ist und auch ein deutlicher Unterschied zu Westdeutschland besteht. 


\begin{tabular}{|l|r|r|r|r|r|r|}
\hline Tabelle 1: Begriffsklassen „Rechts “, 1991 bis 2007 \\
\hline
\end{tabular}

In den alten Bundesländern sollten nach $\mathrm{H} 2 \mathrm{~d}$ Nennungen zur Gesellschaftsordnung und zu wirtschaftlichen Aspekten für Rechts wichtiger sein als in den neuen Ländern. Mit 14 Prozent der Nennungen beziehen sich Westdeutsche mit Assoziationen wie konservativ, stabil etc. doppelt so häufig auf eine „traditionelle Gesellschaftsordnung“. Dass diese in Ostdeutschland nicht so häufig vorkommen, lässt sich möglicherweise damit erklären, dass Konservatismus als Denkfigur in einem den Fortschritt propagierenden System nur wenig Platz hatte. Auch Bezüge zur "kapitalistischen Wirtschaftsordnung“ sind in den neuen Bundesländern mit kürzerer marktwirtschaftlicher Tradition weniger häufig anzutreffen und bestätigen somit die Hypothese.

Da das politische System der Bundesrepublik Deutschland mit seinen Institutionen und Akteuren sich über beide Landesteile erstreckt und gesellschaftlich wichtige Themen über die zum größten Teil bundesweit verbreiteten Leitmedien überall gleich präsent sind, kann 
auch vermutet werden, dass die Gemeinsamkeiten heute ein stärkeres Gewicht haben. Entsprechend formuliert die Hypothese H2e allgemein, dass für die politischen Richtungsbegriffe Ähnlichkeiten zwischen Ost und West bestehen. Für den rechten Pol des Schemas ist allerdings nur wenig Einigkeit festzustellen: Zwar kann das allgemeine Verständnis von Rechts über eine kleine Anzahl von Bedeutungskategorien abgebildet werden, dennoch bestehen Unterschiede im relativen Gewicht der jeweiligen Bedeutungszuschreibungen.

Zusammenfassend ist festzuhalten, dass - während sich die Assoziationen zur rechten Seite des Schemas in Ostdeutschland viel stärker auf einen Bedeutungskern fokussieren - in den alten Bundesländern das Verständnis von Rechts etwas ausdifferenzierter ist.

\subsection{Der Bedeutungsgehalt des politischen Richtungsbegriffes Links in Ost- und Westdeutschland}

Die offenen Nennungen für das Jahr 2007 zeigen, dass mit Links im Allgemeinen themenund gruppenspezifische Inhalte verbunden werden. Vor allem versteht die Bevölkerung darunter Fragen der „sozialen Gerechtigkeit“, die knapp ein Drittel der Nennungen ausmachen (vgl. Tabelle 2). Etwa halb so häufig werden „traditionelle Parteien, Organisationen und Eliten" mit dieser Seite des Schemas assoziiert. Zusammen mit "gesellschaftlichen Gruppen" decken diese Inhalte gut über die Hälfte der Assoziationen ab. Auch für Links zeigt sich damit, dass der Begriff von einer begrenzten Menge an Vorstellungen getragen wird. Aspekte „Neuer Politik“, aber beispielsweise auch Verweise auf „Weltoffenheit“ oder „Internationalismus“ spielen für die Bedeutung von Links nur eine untergeordnete Rolle. Als weitere inhaltliche Aspekte sind noch ,ideologische Bewegungen“ zu nennen. Deren Relevanz, wie auch die der "gesellschaftlichen Gruppen“, unterscheidet sich jedoch in den beiden Landesteilen.

Da die politische Sozialisation im östlichen Teil Deutschlands stark von der Abgrenzungslogik des sozialistischen Regimes geprägt war, sind nach Hypothese H2a deutlich häufigere Nennungen in den Bereichen zu erwarten, die zur eigenen Identitätsbildung und zur Abgrenzung relevante Terminologien beinhalten. Für die linke Seite müssten demnach die Ostdeutschen häufiger „ideologische Bewegungen“ und „sozialistische Wirtschaftsordnung" nennen als die Westdeutschen. Es zeigt sich jedoch, dass beide Kategorien zusammengenommen nicht einmal ein Zehntel aller Nennungen in den neuen Bundesländern ausmachen. Mit zusammen 13 Prozent sind diese im Westen sogar etwas häufiger anzutreffen.

Zentrale Aspekte in der Selbstdarstellung des politischen Systems und vorrangige Staatsziele in der DDR waren die Gleichheit und soziale Sicherheit der Bürger. Auch vor dem Hintergrund der wirtschaftlichen Entwicklung in den Nachwendejahren und der anhaltenden strukturellen Schwäche weiter Teile Ostdeutschlands gehen wir davon aus, dass Verteilungsfragen dort präsenter sind und sich in häufigeren Nennungen mit Bezügen zu „sozialer Gerechtigkeit" niederschlagen (H2b). Diese Argumentation wird auch dadurch gestützt, dass diese Themen in der politischen Kommunikation vor allem durch die Linkspartei, die in den neuen Bundesländern stärker etabliert ist, ständig aktualisiert werden. Tatsächlich verweisen in Ost wie West knapp unter einem Drittel aller Assoziationen mit Links hierauf, so dass zunächst kaum ein Unterschied besteht. Betrachtet man jedoch die mit 13 Prozent in Ostdeutschland nahezu doppelt so häufig vertretenen Nennungen zu „gesellschaftlichen 


\begin{tabular}{|c|c|c|c|c|c|c|}
\hline \multicolumn{7}{|c|}{ Tabelle 2: Begriffsklassen „Links“, 1991 bis 2007} \\
\hline \multirow[b]{2}{*}{ Begriffsklassen: Links } & \multicolumn{3}{|c|}{ D-West } & \multicolumn{3}{|c|}{ D-Ost } \\
\hline & 1991 & $\begin{array}{c}1997 / \\
1998\end{array}$ & 2007 & 1991 & $\begin{array}{c}1997 / \\
1998\end{array}$ & 2007 \\
\hline Ideologische Bewegungen & 20 & 13 & 9 & 18 & 12 & 6 \\
\hline Traditionelle Parteien, Organisationen, Eliten & 9 & 15 & 17 & 6 & 13 & 15 \\
\hline Gesellschaftliche Gruppen & 4 & 10 & 7 & 8 & 16 & 13 \\
\hline Soziale Gerechtigkeit & 16 & 16 & 31 & 19 & 16 & 32 \\
\hline Sozialistische Wirtschaftsordnung & 2 & 4 & 4 & 2 & 3 & 3 \\
\hline Atheismus & 0 & 0 & 0 & 0 & 0 & 0 \\
\hline Utopie & 1 & 1 & 1 & 1 & 1 & 1 \\
\hline Demokratie & 6 & 2 & 1 & 9 & 3 & 2 \\
\hline Internationalismus & 1 & 1 & 1 & 2 & 1 & 2 \\
\hline Weltoffenheit & 8 & 7 & 3 & 9 & 6 & 4 \\
\hline Kritisches Denken / Verhalten & 5 & 2 & 1 & 1 & 2 & 0 \\
\hline „Neue Politik“ & 8 & 9 & 5 & 8 & 4 & 5 \\
\hline Extremismus* & 5 & 6 & 3 & 5 & 5 & 2 \\
\hline Diktatur & 1 & 2 & 2 & 1 & 3 & 2 \\
\hline Parlamentarische Sitzordnung & 3 & 1 & 0 & 1 & 1 & 0 \\
\hline Idiosynkratische Bezüge & 3 & 6 & 5 & 3 & 7 & 6 \\
\hline Sonstiges & 10 & 7 & 11 & 8 & 7 & 8 \\
\hline Prozentsumme aller Nennungen & 100 & 100 & 100 & 100 & 100 & 100 \\
\hline Prozentsumme der Mehrfachnennungen & 156 & 169 & 159 & 158 & 182 & 164 \\
\hline $\mathrm{WN} / \mathrm{KA}$ & 17 & 19 & 36 & 6 & 18 & 25 \\
\hline N-Befragte & 2.554 & 2.335 & 705 & 1.467 & 1.634 & 533 \\
\hline \multicolumn{7}{|c|}{$\begin{array}{l}\text { Anmerkungen: Angaben in Prozent aller gültigen Antworten der Befragten, die den Begriff, „links" defi- } \\
\text { nieren konnten. Addition der einzelnen Werte ergibt die ausgewiesene „Prozentsumme aller Nennun- } \\
\text { gen“. Prozentsumme der Mehrfachnennungen: bei Petra Bauer-Kaase ausgewiesen als „Angaben in Pro- } \\
\text { zent der Befragten, die den Begriff, ,links' definieren konnten“. WN / KA: Anteil aller Befragten (in } \\
\text { Prozent), die auf die offene Frage keine gültige Antwort geben. N-Befragte: Anzahl der Befragten die } \\
\text { mindestens eine Nennung geben konnten. } \\
\text { * Das Codeschema nach Peter Selb fasst unter diese Kategorie auch Nennungen wie „Neonazis“ und } \\
\text { „NPD“, da manche Respondenten bei diesen Zuschreibungen in einer globalen Radikal-Gemäßigt-Di- } \\
\text { mension denken. Vgl. Peter Selb, a.a.O. (Fn. 23), S. } 53 \text {. } \\
\text { Quelle: Die Werte für 1991 wurden übernommen von Peter Selb, a.a.O. (Fn. 23); die für 1997/1998 } \\
\text { von Petra Bauer-Kaase, a.a.O. (Fn. 28). Die Werte für } 2007 \text { stammen aus eigenen Berechnungen von } \\
\text { Daten des DFG-Projekts „Der Einfluss des politischen Vertrauens auf die Unterstützung der Reform des } \\
\text { Sozialstaats in Deutschland“. }\end{array}$} \\
\hline
\end{tabular}

Gruppen“, die Äußerungen wie „die Schwachen“, „der Arbeiter“ oder „der kleine Mann“ beinhalten, so kann man dies doch als einen Hinweis auf eine stärkere Präsenz von Verteilungskonflikten und Deprivationserfahrungen in den neuen Bundesländern interpretieren.

Abschließend bleibt festzuhalten, dass über die linke Seite des Schemas zwischen den beiden Landesteilen eher Einigkeit herrscht, als dass es größere Unterschiede gibt. Dies schlägt sich auch in einer hohen und im Vergleich zu Rechts eindeutig höheren Rangkorrelation von .966 nieder (vgl. Tabelle 3). Die Vermutung, dass die Ostdeutschen sozialisa- 
tionsbedingt häufiger ideologische Aspekte mit Links verbinden, konnte nicht bestätigt werden. Es gibt allerhöchstens Anzeichen dafür, dass in Ostdeutschland der Verteilungskonflikt für die Bürger von größerer Bedeutung für die inhaltliche Struktur von Links ist, denn gesellschaftliche Gruppen wurden dort häufiger genannt. Somit bestätigt sich für den linken Pol des Schemas eher Hypothese H2e, die von Gemeinsamkeiten zwischen den Landesteilen ausgeht.

\subsection{Der Bedeutungswandel der politischen Richtungsbegriffe Links und Rechts}

Als Begriffe, die den politischen Raum strukturieren, müssten Links und Rechts einer bestimmten Dynamik des Wandels unterliegen und gesellschaftliche sowie politische Entwicklungen aufnehmen können. Zum einen stellt sich also die Frage, wie allgemeine gesellschaftliche Veränderungen, die etwa vor dem Hintergrund eines Wertewandels diskutiert werden, von der deutschen Bevölkerung in die Links-Rechts-Dimension integriert werden. Zum anderen ist zwanzig Jahre nach der Wiedervereinigung zu klären, welcher innerdeutschen Wandlungsdynamik das Schema unterliegt - insbesondere vor dem Hintergrund einer möglichen Annäherung oder Auseinanderentwicklung der Antwortstrukturen aus den beiden Landesteilen.

In Anlehnung an Ronald Ingleharts Transformationshypothese wurde in Hypothese H1a formuliert, dass für das Etikett Links bis Mitte der 1990er Jahre die Nennungen zu Aspekten „Neuer Politik“ zunehmen. Gleichzeitig sollten Assoziationen, die in Verbindung mit Verteilungskonflikten stehen, zwar in ihrer inhaltlichen Relevanz abnehmen, aber nicht verdrängt werden. Die stärkere Präsenz und vor allem die deutliche Thematisierung von Fragen der sozialen Gerechtigkeit durch die Eliten seit Mitte der 1990er sollte dagegen nach Hypothese H1b diesen Prozess umkehren: Begriffe im Zusammenhang mit Neuer Politik sollten seit Mitte der 1990er Jahre rückläufig sein, Gerechtigkeitsthemen wieder an Bedeutung gewinnen. In den alten Bundesländern bleibt die Bedeutung von Neuer Politik zwischen 1991 und 1998 auf gleichem Niveau, und Nennungen innerhalb dieser Kategorie wie Umweltorientierung, Gleichberechtigung und Ausländerfreundlichkeit halbieren sich im Laufe der nächsten zehn Jahre knapp. In Ostdeutschland liegen die Werte nach 1991 ständig bei circa fünf Prozent (vgl. Tabelle 2).

Die Nennung ,soziale Gerechtigkeit“ nimmt über den gesamten Zeitraum in beiden Landesteilen deutlich zu. Im Einklang mit der Hypothese H1a gibt es aber in Ostdeutschland zwischen 1991 und 1997 einen leichten Rückgang der Nennungen, in Westdeutschland bleiben diese zu beiden Zeitpunkten auf gleichem Niveau. Zum großen Sprung von Themen „sozialer Gerechtigkeit“ für die inhaltliche Bedeutung von Links kommt es in der Phase zwischen den beiden jüngsten Erhebungszeitpunkten, in der sich die Zahlen in beiden Teilen Deutschlands auf knapp ein Drittel aller Nennungen verdoppeln. Einschränkend ist anzumerken, dass aufgrund des Untersuchungsthemas „Vertrauen und Wohlfahrtsstaat“ 2007 Aspekte sozialer Gerechtigkeit den Befragten während der Umfrage häufig begegnen - diese Themen also in hohem Maße zugänglich sind. ${ }^{65}$ Bei vorsichtiger Interpretation kann man konstatieren, dass der Verteilungskonflikt an Bedeutung gewinnt. Zusam- 
men mit dem Befund, dass Themen Neuer Politik an Bedeutung verlieren, in den neuen sogar früher als in den alten Bundesländern, deckt sich diese Entwicklung mit den Annahmen aus Hypothese H1b. ${ }^{66}$

Eine ähnliche Entwicklung lässt sich auch für die rechte Seite des Schemas vermuten. So sollten in den Bedeutungszuschreibungen zunächst wirtschaftsbezogene Äußerungen weniger wichtig werden und Aspekte der „traditionellen Gesellschaftsordnung “ an Bedeutung gewinnen (H1c), bevor sich diese Entwicklung ab Mitte der 1990er Jahre wieder umkehrt (H1d). Die Zahlen in Tabelle 1 bestätigen die Hypothesen nur zum Teil und deuten auf Unterschiede zwischen den beiden Landesteilen. So lässt sich zwar keine Zunahme der Nennungen zur „traditionellen Gesellschaftsordnung“ zwischen den ersten beiden Erhebungszeitpunkten erkennen, doch entfallen darauf bereits 1991 die mit Abstand meisten in Ost wie West. In der weiteren Entwicklung gehen die Nennungen dieser Kategorie beiderseits kontinuierlich zurück, in den neuen Bundesländern ist dieser Effekt jedoch deutlich stärker ausgeprägt. Die in Hypothese H1d vermutete Bedeutungszunahme von Themen der Wirtschaftsordnung und der Eigenverantwortung Anfang der 1990er Jahre zeigt sich nur minimal in zunehmenden Assoziationen von Rechts mit „Egoismus“ (unter anderem "unsozial“, „Ungleichheit"), nicht jedoch bei der „kapitalistischen Wirtschaftsordnung“, die zumindest in Ostdeutschland stetig an Relevanz verliert. Möglicherweise bleibt die Revitalisierung wirtschaftlicher Themen im rechten Bedeutungsspektrum aufgrund der Umdeutung des Etiketts hin zu rechtsextremen Inhalten aus.

Als eine weitere Entwicklung, die sich auf das inhaltliche Verständnis der beiden Richtungsbegriffe auswirkt, muss das über den Mauerfall symbolisierte Ende der Auseinandersetzung zweier ideologischer Systemalternativen berücksichtigt werden. Hypothese H1e erwartet hierzu in beiden Teilen Deutschlands auf der linken Seite des Schemas einen Rückgang von Assoziationen, die sich auf ,ideologische Bewegungen“ wie Sozialismus und Kommunismus beziehen, für die rechte Seite eine Abnahme von Nennungen mit Bezug zur „kapitalistischen Wirtschaftsordnung“. Während 1991 in Westdeutschland noch jede fünfte Nennung darauf entfällt, verlieren „ideologische Bewegungen“ immer weiter an Bedeutung und beinhalten 2007 nur noch neun Prozent der Antworten. Auch in Ostdeutschland, wo zum ersten Datenpunkt noch ein Viertel der Nennungen auf diese Kategorie entfallen, sinkt die Relevanz von ideologiebezogenen Assoziationen. Die Entwicklungen für Rechts bei Äußerungen zur „kapitalistischen Wirtschaftsordnung“ verlaufen jedoch uneinheitlich. So liegen die Nennungen in Westdeutschland, abgesehen vom ersten Erhebungszeitpunkt, auf ähnlichem Niveau um zehn Prozent. In Ostdeutschland nehmen Verweise auf Aspekte der freien Marktwirtschaft aber nach den hohen Werten im Jahr 1991 um mehr als die Hälfte ab. Zwar verlieren „ideologische Bewegungen“ an Bedeutung, aber zumindest in der westdeutschen Bevölkerung sind mit knapp jeder zehnten Nennung Aspekte der „kapitalistischen Wirtschaftsordnung" auch nach dem Mauerfall immer noch wichtig.

Die Hypothesen zum allgemeinen Wandel der Inhalte des Links-Rechts-Schemas haben sich nur zum Teil bestätigt. Es zeigte sich aber, dass das Schema neue Themen und Konflikte in der politischen Landschaft aufnehmen kann, überholte Inhalte jedoch auch an Bedeutung verlieren können.

66 Hinzu kommt, dass die Nennungen zu verwandten Themen wie „Weltoffenheit“ oder „Internationalismus" ebenfalls zurückgehen oder auf niedrigstem Niveau stagnieren. 
Zwischen den Bürgern der alten und neuen Bundesländer besteht also zwanzig Jahre nach dem Mauerfall zumindest für Links relative Einigkeit über die inhaltliche Bedeutung, und das Schema konnte sich in beiden Landesteilen gleichermaßen an gesellschaftliche Entwicklungen wie das Wiedererstarken von Verteilungsfragen zu Lasten von Themen Neuer Politik anpassen. Nun stellt sich die Frage, wie die Dynamik des Wandels im Begriffsverständnis von Links und Rechts im Ost-West-Vergleich zueinander verlaufen ist. Gemeinsamkeiten in den Bedeutungszuschreibungen und deren Veränderungen können die Folge von Annäherungen ( $\mathrm{H} 3 \mathrm{a})$, aber auch von parallelen Entwicklungen sein $(\mathrm{H} 3 \mathrm{~b})$, je nachdem, wie ähnlich die Vorstellungen von den Inhalten des Links-Rechts-Schemas nach dem Mauerfall waren. Unterschiede können analog dazu die Folge paralleler Entwicklung sein, wenn sich beide Landesteile bereits zu Beginn der Zeitreihe unterscheiden (H3c) oder sich als Folge eines Auseinanderdriftens der Bedeutungszuschreibungen darstellen (H3d). In Tabelle 3 sind die Rangkorrelationen zwischen Ost- und Westdeutschen je Erhebungszeitpunkt jeweils für Links und Rechts ausgewiesen. Geht man von einer Annäherung aus, so sollten die Koeffizienten zwischen Ost und West zu Beginn der Zeitreihe geringer sein und stetig zunehmen (umgekehrt bei der Auseinanderentwicklung). Bei einem parallelen Verlauf müssten die Werte auf gleichem Niveau bleiben, das umso höher liegt, je ähnlicher sich beide Landesteile bereits nach dem Mauerfall waren.

\begin{tabular}{|c|c|c|c|c|c|c|c|c|c|}
\hline \multicolumn{10}{|c|}{ Tabelle 3: Rangkorrelationen Rechts und Links in Ost und West } \\
\hline \multicolumn{5}{|c|}{ Rangkorrelationen Rechts } & \multicolumn{5}{|c|}{ Rangkorrelationen Links } \\
\hline & & & Ost & & & & & Ost & \\
\hline & & 1991 & 1997 & 2007 & & & 1991 & 1997 & 2007 \\
\hline \multirow{3}{*}{ West } & 1991 & $.828^{* *}$ & & & & 1991 & $.813^{* *}$ & & \\
\hline & 1997 & & $.913^{* *}$ & & West & 1997 & & $.949^{* *}$ & \\
\hline & 2007 & & & $.834^{* *}$ & & 2007 & & & $.966^{* *}$ \\
\hline \multicolumn{10}{|c|}{$\begin{array}{l}\text { Anmerkung: Koeffizient: Spearman's rho; }{ }^{*} \mathrm{p}<.05,{ }^{* *} \mathrm{p}<.01 \text {; Rangkorrelationskoeffizient: Verhältnis } \\
\text { zwischen den Rängen der Bedeutungskategorien. } \\
\text { Quelle: Eigene Berechnungen; vgl. Anmerkungen zu Tabelle } 1 \text { und } 2 .\end{array}$} \\
\hline
\end{tabular}

Auf der rechten Seite des Schemas zeigt sich, dass die Bürger in den alten und neuen Bundesländern zum ersten gemeinsamen Erhebungszeitpunkt schon recht ähnliche Vorstellungen davon hatten, was Rechts bedeutet. Nach einer Annäherung 1997 entwickelte sich das inhaltliche Verständnis jedoch wieder auseinander (vgl. H3d). Während zwischen 1991 und 1997 die inhaltliche Struktur in beiden Landesteilen einem Wandel unterworfen ist, zeigt sich im Vergleich zwischen den Erhebungszeitpunkten 1997 und 2007 für Westdeutschland in der Rangkorrelation eine deutlich geringere Fluktuation als für Ostdeutschland (tabellarisch nicht ausgewiesen).

Anders verhält es sich auf der linken Seite des Schemas. Auch hier herrscht zu Beginn zwischen Ost- und Westdeutschen relative Einigkeit in den Bedeutungszuschreibungen. Allerdings nähert sich das Verständnis von Links über die Zeit weiter an, so dass hierbei aktuell eine große Übereinstimmung besteht. Unterstützt wird dieser Befund auch dadurch, dass zwischen den Erhebungszeitpunkten mit zunehmender Aktualität die semantische Bedeutung immer geringeren Schwankungen unterliegt. Für die linke Seite des Schemas lässt sich somit eindeutig eine Annäherung zwischen den beiden Landesteilen konstatieren, die verantwortlich ist für die Befunde zum aktuellen Bedeutungsgehalt. 
Die zwei Seiten des Schemas folgen demnach unterschiedlichen Entwicklungslogiken. Zwar kommt es in den ersten Jahren nach dem Mauerfall zu einer Annäherung von Ost und West (H3a). Diese ist aufgrund von großen Systemunterschieden vor der Wiedervereinigung und einer Orientierungsphase ostdeutscher Bürger bei der Nutzung des Schemas im gesamtdeutschen Kontext durchaus plausibel. Vergleicht man die Dynamiken der beiden Richtungsbegriffe im weiteren Verlauf, lässt sich folgendes feststellen: Während sich für Links ein gemeinsames Verständnis festigt, gehen die Deutungen für Rechts wieder auseinander. Dies ist vor allem vor dem Hintergrund zu verstehen, dass sich speziell in Ostdeutschland im Wandel des Etiketts Rechts eine Entwicklung widerspiegelt, bei der Kategorien wie traditionelle Akteure und Gesellschaftsbilder unter dem Aufkommen rechtsextremer Assoziationen an Bedeutung verlieren.

\section{Links-Rechts: Flexible Interpretationen, unterschiedliche Logiken des Wandels}

Die Vorstellungen der Ost- und Westdeutschen über Rechts und Links weisen heute insofern Gemeinsamkeiten auf, als im gesamten Bundesgebiet einige wenige Bedeutungskategorien einen Großteil des Begriffsverständnisses abbilden können. Hinzu kommt, dass über die linke Seite des Schemas relative Einigkeit herrscht. Klare Unterschiede in den Ergebnissen aus den alten und neuen Bundesländern wurden jedoch auf der rechten Seite des Schemas deutlich, wo das Bedeutungsspektrum eher unterschiedlich strukturiert ist. Während sich die Aussagen der Ostdeutschen zu Rechts stark auf Aspekte von Extremismus konzentrieren, lässt sich im alten Bundesgebiet ein heterogeneres Bild zeichnen.

Vor allem der politische Richtungsbegriff Links hat eine wesentliche Umdeutung erfahren - von einem Verständnis, das vor allem von der Auseinandersetzung mit der Ideologie des Ostblocks geprägt war, hin zu einem von Fragen der Verteilung und der Gerechtigkeit bestimmten Begriff. Auch das Verständnis von Rechts wandelt sich - allerdings, mit Ausnahme des Bedeutungszuwachses von Extremismus, ohne erkennbaren Trend.

Hinsichtlich eines Wandels im Begriffsverständnis von Ost- und Westdeutschen ist hervorzuheben, dass die beiden Pole einer unterschiedlichen Dynamik folgen: Während für Rechts keine eindeutige Entwicklung festzustellen war, konnte für den linken Pol eine eindeutige Annäherung ausgemacht werden.

Diese Ergebnisse für Deutschland unterstreichen, dass Interpretationen des LinksRechts-Schemas flexibel sind und Reaktionen auf gesellschaftliche Entwicklungen darstellen. Zudem hat sich gezeigt, dass Links und Rechts in der deutschen Bevölkerung unterschiedlichen Logiken des Wandels folgen. Dies spricht dafür, in weiteren Analysen auf Unterschiede zwischen der linken und der rechten Seite des Schemas zu achten, gerade wenn es um dessen Nutzung in der politischen Kommunikation und der individuellen Meinungsbildung geht. 